\title{
An Empirical Study of Note Taking and Task Repetition on Noticing and EFL Writing
}

\author{
Ling Shi ${ }^{1, a}$, Xin Yuan ${ }^{2, b}$, Min Wu ${ }^{3, c}$, Zuhong Lu ${ }^{4, d}$, Yangyang Liu ${ }^{5, e}$ \\ ${ }^{1}$ Southeast University, No. 2, Sipailou, Nanjing, 210096, Jiangsu Province, China \\ 2 Nanjing Pujiang Institute, No. 96, Tangfenglu, 211134, Jiangsu Province, China \\ ${ }^{3}$ Southeast University, No. 2, Sipailou, Nanjing, 210096, Jiangsu Province, China \\ ${ }^{4}$ Southeast University, No. 2, Sipailou, Nanjing, 210096, Jiangsu Province, China \\ ${ }^{5}$ Nanjing University, No. 22, Hankoulu, Nanjing, 210093, Jiangsu Province, China \\ a shelleyshil@126.comb458612967@qq.com cwumin77131@aliyun.com \\ dzhlu@seu.edu.cne116959252@qq.com
}

\begin{abstract}
Keywords: Note taking; Task Repetition; Noticing; Output; EFL Writing
Abstract. This paper set out to probe into the learning strategies that help promote Chinese college students' noticing and memory retention of targeted language through the mental process of input into intake. Three experimental groups-Group A, the control group (without note taking and task repetition), Group B-experimental group (with note taking and non-task repetition) and Group C-experimental group (with both note taking and task repetition) were involved. Through a five-stage writing task consisting of output production, comparison to the model text, writing rehearsal and two revisions (immediate rewriting and delayed rewriting), the paper examined what language features Chinese college students have noticed by taking notes, and how such noticing affected short-term and long-term memory retention. It was found that $80 \%$ language features that participants noticed in production, comparison and revision stage was lexical features; the multiple comparisons of writings in stage 3 revealed significant differences between Group A and Group B ( $\mathrm{p}=0.002)$, Group A and Group C $(\mathrm{p}<0.001)$, but no significant differences found between Group B and Group $\mathrm{C}(\mathrm{p}>0.05)$. The results indicate that note taking and task repetition help improve students' short-term and long-term memory.
\end{abstract}

\section{Introduction}

According to Schmidt ${ }^{[1]}$, "learning without attention to what is to be learned is impossible". By proposing Noticing Hypothesis, Schmidt starts from cognitive psychology perspective to examine the effect of noticing ${ }^{[2][3]}$. Swain ${ }^{[4]}$, after raising Output Hypothesis, began to study the noticing triggered from doing output tasks. However, little research has been done to investigate the noticing function of output, particular in the context of learning English as a foreign language (EFL) like China. Therefore, this study aims to examine the function of note taking and task repetition in facilitating noticing and longer memory retention in comparison to the think-aloud protocol used in non-note taking group. The research has basically three research goals: The first was to investigate the effects of note taking and task repetition on the noticing of foreign language forms; the second focused on how noticing facilitated foreign language acquisition in both short-term and long-term learning effect and the third one was concerned with the impact of note-taking and task repetition on Chinese college students' writing performance.

\section{Research questions}

Five questions will be answered in the research.

1) In doing the initial description task, what types of language features do most English learners notice?

2) By comparing and rewriting compositions, what language features do English learners notice and intake from the provided model? 
3) What are the effects of note-taking on short-term retention of target forms in the immediate rewriting stage?

4) Does giving students one more chance to do the rewriting within a short time span improve their writing in the delayed-rewrite task?

5) What are the long-term effects of note-taking and task repetition on the foreign language learners?

\section{Materials \& Methodology}

1. Participants \& Materials.Participants were sophomores with the same language background (Chinese) in the Southeast University, China. All of them were non-English majors from three classes, and all had passed CET 4 which would ensure the approximate English proficiency. Group A was the control group. Group B and Group C were the experimental groups. The research settled on the evaluation of the writing performance of the three groups.

To exclude the influence brought by individual language ability, a reading test was given as the measurement of the pre-experiment English proficiency. The pre-test reading was taken before the five-stage task in about 20 minutes. The test was all single-choice questions. The reading passage was selected from TOEFL-ibt reading test with 12 questions. Those whose scored above 6 were allowed to participate in the experiment.

2. Procedure. Participants completed a five-stage task, similar to the experiment carried out by Eun Young-Kang ${ }^{[5]}$ :

a) To write a picture description;

b) To compare it with a model text written by an English native speaker;

c) To rewrite the original description without reference to the model text;

d) To repeat the writing task, that is, to revise the text after one-week interval;

e) To have an unannounced delayed-rewrite-task after a two-week interval.

The experiment went during the regular English classes. Picture description writing task was used to have learners notice the gaps/holes in their inter-language knowledge. In the first composing stage, Group A was given Sheet 1, Group B and C with Sheet 1 and Sheet 2 (one for writing task and one for notetaking). All the three groups were asked to write a 120 -word description in response to a picture prompt on Sheet 1. During the process of describing picture, Group B and C had to jot down on Sheet 2 the difficulties and problems they encountered. Participants were allowed 15 minutes to finish the writing task in stage1. Immediately after this, they were given a piece of native speaker model text.

In stage 2, Group B and C received Sheet 3 (the same to Sheet 1 ) and all the paarticipants could have one native-speaker model text. They spent 10 minutes reading through the model and made comparisons with their original writing, but only Group B and $\mathrm{C}$ were allowed to jot down whatever language features they noticed in the model (i.e. useful lexical forms or structures). Meanwhile, Group A used only think-aloud protocol to make comparison. At the end of this stage, both the models and the sheets were collected except the pictures because it would be used in the following stage.

In stage 3, students were asked to rewrite immediately upon referring to the reading text on Sheet 4 in 15 minutes. And in stage 4, after one-week interval, Group C was asked to rewrite the initial writing based on the same picture on Sheet 5 in 15 minutes.

The delayed-rewriting task was conducted after two-week interval without an announcement. In this stage, all the groups received Sheet 6 and were asked to rewrite the description in 15 minutes.

3. Methods. h Hanaoka ${ }^{[6]}$ and Qi and Lapkin ${ }^{[7]}$ adopt the classification of language-related episodes proposed by William ${ }^{[8]}$. In this study, the problems and difficulties of picture description in the first stage were named as problematic features noticed in stage 1 and the features noticed in the following stages. To investigate the types of language features students have noticed, the research also classified the language difficulties and problems that the participants had taken down on sheets of notes into four categories: lexis, grammar, content, and other. This paper used the statistical package for the Social science (SPSS20), with the alpha level set at .05, to check the performance of the three groups. 


\section{Results \&Discussion}

1. Noticed Language Features During Output and Input Activities. The first question concerned with the types of language features that participants noticed in the first writing task of stage 1 . The frequencies and ratios of problematicfeatures that the participants recognized were shown in table 1 . Participants recognized a total of 106 problematic features including 86 lexis items, 4 grammar problems, 9 content problems and 7 other issues concerning expressions about feelings or preferences. The percentage of the noticed lexical items of Group A was 82.9\%, Group B was 74.3\%, and Group C was $86.1 \%$ (see table 1). Thus, the noticed problematic language features in the first stage were overwhelmingly lexical.

Table 1: Frequencies and Ratios of Language Features Noticed in stage 1

\begin{tabular}{lllllllllllllllll}
\hline & Group A & $(\mathrm{n}=12)$ & \multicolumn{4}{c}{ Group B $(\mathrm{n}=12)$} & \multicolumn{4}{c}{ Group C $(\mathrm{n}=14)$} & \multicolumn{4}{c}{ All Participants (n=38) } \\
\hline & $\mathrm{N}$ & $\%$ & $\mathrm{M}$ & $\mathrm{SD}$ & $\mathrm{N}$ & $\%$ & $\mathrm{M}$ & $\mathrm{SD}$ & $\mathrm{N}$ & $\%$ & $\mathrm{M}$ & $\mathrm{SD}$ & $\mathrm{N}$ & $\%$ & $\mathrm{M}$ & $\mathrm{SD}$ \\
Lexis & 29 & 82.9 & 2.4 & 1.2 & 26 & 74.3 & 2.2 & 1.8 & 31 & 86.1 & 2.2 & 0.6 & 86 & 81.1 & 2.3 & 1.2 \\
Grammar & 2 & 5.7 & 0.2 & 0.4 & 2 & 5.7 & 0.2 & 0.2 & 0 & 0.0 & 0.0 & 0.0 & 4 & 3.7 & 0.1 & 0.1 \\
Content & 3 & 8.6 & 0.3 & 0.5 & 3 & 8.6 & 0.3 & 0.2 & 3 & 8.3 & 0.2 & 0.2 & 9 & 8.5 & 0.2 & 0.2 \\
Other & 1 & 2.9 & 0.1 & 0.3 & 4 & 11.4 & 0.3 & 0.2 & 2 & 5.6 & 0.1 & 0.1 & 7 & 6.7 & 0.2 & 0.2 \\
Total & 35 & 100 & 3.0 & 1.5 & 35 & 100 & 3.0 & 2.2 & 36 & 100 & 2.5 & 1.0 & 106 & 100 & 2.8 & 1.7 \\
\hline
\end{tabular}

The second question addressed the types of language features that participants noticed from a native-speaker model text and then incorporated them into their revision production. The results showed that learners in all three groups inserted more lexical items than language structures or other issues from the model text. Group A adopted a total of 59 linguistic features, of which 50 concerned lexical items. Group B, similarly, adopted 93 linguistic items, in which vocabulary accounted for 79. Group C with their vocabulary amounting to 98 (see figure1), showed even stronger inclinations. In all, the type of incorporated language features in stage 2 was also lexical oriented.

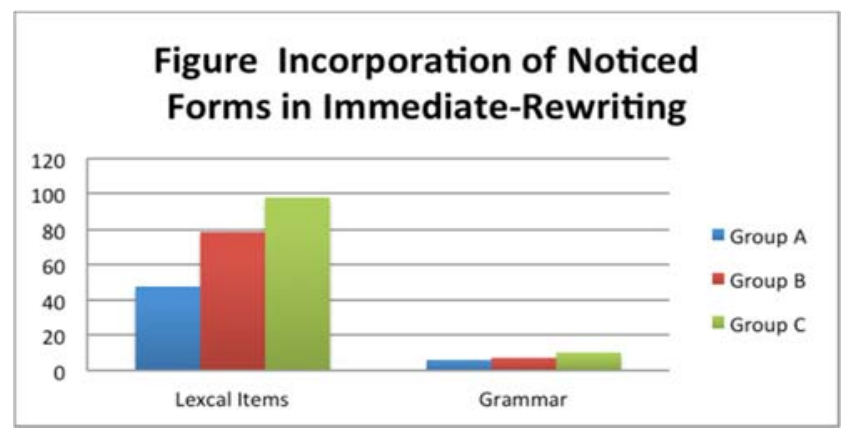

Figure 1. Incorporation of noticed forms in immediate-rewriting

2. Noticing Function in the Output and Input Activities. The third question focused on the influence of note taking on learners' noticing and incorporation of language features from the given text or to examine the effect of note taking on short-term memory retention. Every incorporated target language feature was compared and analyzed between the three groups (see table 2). The results showed that each participant in Group B included 7.75 noticed language features and Group $\mathrm{C}$ was 8.00 while Group A included only 4.92 noticed language features. To verify the significance of the difference between note taking group and non-note taking groups, One-way ANOVA test was adopted to exam the mean scores. Table 2 indicatedthe significant difference between Group A and Group B ( $p=0.002)$, Group A and Group C $(p<0.001)$ in their immediate rewriting stage. No significance difference was found between Group B and Group C ( $>>0.05)$. 
Table 2: Variance analysis of incorporation of language features in immediate rewriting

\begin{tabular}{lcccc}
\hline Group(I) & Group(J) & Mean Difference (I-J) & Std.Error & Sig. \\
\hline $\mathrm{A}$ & $\mathrm{B}$ & $-2.83333^{*}$ & .71437 & .002 \\
& $\mathrm{C}$ & $-3.08333^{*}$ & .68838 & .000 \\
$\mathrm{~B}$ & $\mathrm{~A}$ & $2.83333^{*}$ & .71437 & .002 \\
& $\mathrm{C}$ & -.25000 & .68838 & .936 \\
$\mathrm{C}$ & $\mathrm{A}$ & $3.08333^{*}$ & .68838 & .000 \\
& $\mathrm{~B}$ & .25000 & .68838 & .936 \\
\hline
\end{tabular}

*. The mean difference is significant at the 0.05 level.

It was found that when learners were motivated to find out the correct expressions in order to well describe the picture, their noticing function was activated which increased their attention on the following comparison task. Participants all felt a strong need to read as carefully as possible the model text and a great desire to incorporate the noticed words and expressions, or even the whole structure of the relevant input model in their revisions for they knew that those linguistic items represent a better or more native way to describe the picture.

3. Effects of NoteTaking on Memory. To answer question 4, Group C was added to constitute a comparison to Group B for the purpose of examing the effects of note taking on memory. To be specific, Group $\mathrm{C}$ was given one more chance to do the delayed rewriting task one week later after the initial description task.

All the participants in the three groups had a final unnoticed delayed-rewrite task after two-week interval. The figures in Table 3 were the results of Group A, Group B and Group C in the two-week-delayed rewriting task. (see table 3)

Table 3 Variance analysis of incorporation of language features in two-week delayed rewriting

\begin{tabular}{lllll}
\hline Group (I) & Group (J) & Mean Difference (I-J) & Std. Error & Sig. \\
\hline \multirow{2}{*}{$\mathrm{A}$} & $\mathrm{B}$ & $-2.333^{*}$ & .638 & .003 \\
& $\mathrm{C}$ & $-3.929^{*}$ & .615 & .000 \\
$\mathrm{~B}$ & $\mathrm{~A}$ & $2.333^{*}$ & .638 & .003 \\
& $\mathrm{C}$ & $-1.595^{*}$ & .615 & .046 \\
$\mathrm{C}$ & $\mathrm{A}$ & $3.929^{*}$ & .615 & .000 \\
& $\mathrm{~B}$ & $1.595^{*}$ & .615 & .046 \\
\hline
\end{tabular}

*. The mean difference is significant at the 0.05 level.

The mean score of language features incorporated in the final stage rewriting in Group A was 2.00, Group B was 4.33, and Group C was 5.93. The results from the table indicated that task repetition has significant impacts on long-time memory retention $(p<0.05)$. As far as $E F L$ is concerned, task repetition could be considered as a crucial strategy for strengthening the long-term retention in the process of language learning.

The findings in this research confirm that the effect of note taking on language development is important. By comparing notes and essays of Group A, Group B and Group C, we found that note taking is an effective strategy to better language learning because it can help learners notice the gap between the models and their owns. With the help of the model input, learners tend to insert linguistic items contained in the native speaker input model as they had jotted down and to store the linguistic items which they found useful in their long-term memory system. That participants incorporate more language features in the two following rewriting tasks suggested that notes, to some extent, facilitated the understanding of new language features and its retention. One plausible explanation was that note taking activity was related to their mental cognition processing and exerted positive effects on language learning.

4. Effects of Task Repetition on Memory. Group $C$ was chosen to have one more chance to rewrite the picture description after one week interval. Their performance of production in the stage 5 confirmed that task-repetition helped students apply more language features from the model text in their delayed-rewriting test. Task repetition was a student-oriented way of teaching and learning foreign 
language. Instead of teacher's guiding them on how to describe the picture, students found their own problems and mistakes in their initial production by the help of the native speaker's model writing, which createed needs and motivation to read, rememberand store the relevant model input in their minds.

5. Long-term Effects of Note Taking and Task Repetition on Memory. To examine the effects of note taking on long-term memory retention, we compared the incorporated language features in two-week delayed output production written by Group A, Group B and Group C. The figures in Table 3 found obvious significance using note taking as a strategy to facilitate long-term retention $(p=0.003)$. By comparing the results of Group A with Group C, significant difference could found. $(p<0.001)$. It was clear that participants in Group $C$ incorporated and memorized language features best in the three groups, which confirms the hypothesis that note taking and task repetition allow students to be more familiar with the new language features they came across in the stage 1 and stage 2, and facilitates the storing of native lexical items and structures into long-term memory retention.

\section{Conclusion}

Note taking and task repetition are proven to be effective to facilitate the storing of information into short-term and long-term memory, which may add to traditional way of teaching writing through the method of a configuration of teacher and peer feedback. Also, the findings in this study may carry implications for other EFL teaching and learning.

\section{References}

[1] Schmidt, R. Implicit learning and the cognitive unconscious. In N. Ellis (Eds), Implicit and explicit learning of languages. London: Academic Press (1994a), p.165-209.

[2] Schmidt, R. Deconstructing consciousness in search of useful definitions for applied linguistics. AILA Review 11, (1994b), p.11-26.

[3] Schmidt, R. Attention, awareness, and individual differences in language learning. In W. M. Chan \& S. et. al [Eds], Proceedings of CLaSIC. Singapore, December 2-4, (2010), p. 721-737.

[4] Swain, M. Output Hypothesis: Its History and Its Future. Foreign Language Teaching and Research Vol. 40, No.1, (2008).

[5] Eun Young-Kang. Effects of output and note-taking on noticing and inter-language development. Columbia University Working Papers in TESOL \& Applied Linguistics, Vol. 10, No. 2, (2010).

[6] Hanaoka, O. Output, noticing, and learning: An investigation into the role of spontaneous attention to form in a four-stage writing task. Language Teaching Research, 11, (2007), p. 459-479.

[7] Qi, D.S. and Lapkin, S. Exploring the role of noticing in a three-stage second language writing task. Journal of Second Language Writing, 10. (2001), p. 277-303.

[8] Williams, J. Learner-generated attention to form. In Ellis, R. [Eds], Form-focused instruction and second language learning. Malden, MA: Blackwell, (2001), p. 303-46. 